\title{
Embarazo abdominal: análisis de una "epidemia"
}

\author{
Eduardo León Jaramillo V**
}

\begin{abstract}
RESUMEN: Se analiza el "comportamiento epidémico" de embarazo abdominal en mujeres atendidas en el Hospital de Caldas, Manizales, Colombia, entre 1993 y 1995 . Se presenta un resumen de 4 casos y se revisa la literatura sobre el tema destacando aspectos epidemiológicos, clínicos y de manejo.
\end{abstract}

PALABRAS CLAVES: Embarazo ectópico, embarazo abdominal.

SUMMARY: An "epidemic" of abdominal pregnancy in women attended at Hospital de Caldas, Manizales, Colombia, between 1993 and 1995 is reported. Four women with abdominal pregnancy are informed and the literature is reviewed highlighting epidemiologic, clinic and management aspects.

KEY WORDS: Ectopic pregnancy, abdominal pregnancy.

\section{Introducción}

Un embarazo en el cual el feto se desarrolla en las superficies peritoneales del abdomen se denomina embarazo abdominal. Entre 1993 y 1995 se diagnosticaron en el Hospital de Caldas (HC), Manizales, Colombia, 6 embarazos abdominales, lo cual constituye una "epidemia" si se tiene en cuenta que desde 1975 no había ocurrido ningún caso. El primero de la serie lo publicamos en la Revista Colombiana de Obstetricia y Ginecología con un análisis de la incidencia de embarazo ectópico desde 1970 (1). En el presente artículo hago un análisis del "comportamiento epidémico" de embarazo abdominal en el HC y reviso aspectos clínicos y de manejo, y analizo la mortalidad materna y perinatal resultante de esta forma anormal de gestación.

\section{Materiales y métodos}

El HC, situado en Manizales, Colombia, es un hospital de enseñanza, de tercer nivel de atención. Para la elaboración del presente trabajo, consulté la base de datos computarizada disponible en la sección de estadísticas del hospital, entre 1993 y 1995 . Identifiqué 229 pacientes con diagnóstico de embarazo ectópico, de los cuales seleccioné 6 con embarazo abdominal. Encontré en el archivo clínico 5 de las 6 historias clínicas. De los 5 casos revisados, uno ya fue publicado (1) y, por tanto, lo excluyo del presente informe. En tablas presento las características de las mujeres, los hallazgos ecográficos y quirúrgicos y las analizo en su conjunto destacando los hallazgos más importantes y analizo los aspectos clínicos y epidemiológicos del embarazo abdominal, a la luz de la literatura sobre el tema.

\section{Resultados}

Durante el período 1993-95 se diagnosticaron en el HC, $6(2,5 \%)$ embarazos abdominales de un total de 229 mujeres con embarazo ectópico y ocurrieron 12,961 nacimientos vivos para una tasa de 4.6 embarazos abdominales por 100.000 nacidos vivos.

De los 4 casos aquí referidos, 2 fueron embarazos abdominales primarios y los otros 2 fueron secundarios a embarazos tubáricos.

Todas eran mujeres fértiles, con ciclos menstruales regulares, sin antecedentes quirúrgicos ni expuestas a factores de riesgo para embarazo ectópico, salvo en el caso I que tenía antecedentes de ingesta de anticonceptivos orales ( $\mathrm{ACO}$ ) durante 5 años y en el caso 4 a quien se le identificó una VDRL reactiva durante el primer trimestre del embarazo (Tabla 1).

El dolor abdominal fue el síntoma más prevalente en todas las mujeres, con varias semanas de evolución, salvo en el caso 4 que cursó asintomática hasta 24 horas antes de consultar. Sólo una paciente presentó historia de sangrado genital durante el embarazo. En los tres primeros casos el cérvix estaba cerrado y en el cuarto no fue posible palpar el cérvix (Tabla 1).

Las dos pacientes con embarazo temprano presentaron un cuadro clínico de choque hipovolémico y abdomen agudo (Tabla 1) que requirió cirugía urgente que condujo al diagnóstico; en una de ellas el diagnóstico prequirúrgico fue apendicitis aguda y en la otra se pensó en un embarazo ectópico roto o en quiste torcido de ovario como diagnóstico diferencial; en ambas se encontró hemoperitoneo abundante proveniente de sangrado del sitio de implantación placentaria. Cabe destacar que en las ecografías realizadas a estas pacientes, no se informó sobre líquido libre en cavidad abdominal (Tabla 2). 
Tabla 1

CARACTERISTICAS DE LAS MUJERES CON EMBARAZOABDOMINAL

\begin{tabular}{|lllll|}
\hline \multicolumn{5}{c|}{ Casos de embarazo abdominal } \\
\hline Variable & $\mathbf{1}$ & $\mathbf{2}$ & $\mathbf{3}$ & $\mathbf{4}$ \\
\hline Fecha & $3-02-94$ & $4-10-94$ & $24-05-95$ & $9-09-95$ \\
Procedencia & Supía & Manizales & Riosucio & Manizales \\
Edad (años) & 33 & 30 & 29 & 38 \\
Gravidez & 1 & 4 & 1 & 3 \\
Partos & 0 & 3 & 0 & 1 \\
Abortos & 0 & 0 & 0 & 1 \\
Gestación (semanas) & 41 & 15 & 14 & 42 \\
VDRL Reactiva & - & - & - & + \\
ACO & + & - & - & - \\
Dolor abdominal & + & + & + & + \\
Abdomen agudo & - & + & + & - \\
Homalgia & - & + & - & - \\
Sangrado genital & - & - & + & - \\
Cérvix & Cerrado & Cerrado & Cerrado & No palpable \\
\hline
\end{tabular}

Tabla 2

ECOGRAFIAS DE LAS MUJERES CON EMBARAZO ABDOMINAL

\begin{tabular}{|lllll|}
\hline \multicolumn{5}{c|}{ Casos de embarazo abdominal } \\
\hline Ecografía & $\mathbf{1}$ & $\mathbf{2}$ & $\mathbf{3}$ & $\mathbf{4}$ \\
\hline Feto & Vivo & Vivo & Vivo & Vivo \\
Localización & In útero & In útero & S.D. & Abdominal? \\
Situac./Presentac. & Transv. & S.D. & S.D. & Pelvis \\
Líquido amniótico & Oligoh. & S.D. & Oligoh. & Oligoh. \\
Placenta & Previa & S.D. & S.D. & Previa \\
Líquido libre & S.D. & S.D. & S.D. & S.D. \\
\hline
\end{tabular}

La ecografía no contribuyó con el diagnóstico de embarazo abdominal en ninguno de los casos, aún cuando en el caso 4 se sospechó este diagnóstico (Tabla 2).

En las pacientes con embarazo de término, el diagnóstico ecográfico fue de feto vivo en situación transversa (caso 1) y presentación de pelvis (caso 4), oligohidramnios severo y placenta previa total, en ambos casos (Tabla 2).

La implantación placentaria fue exclusivamente en la serosa del fondo uterino, en el caso 1; en el 2 fue en el anexo derecho; en el 3, involucraba el anexo izquierdo, el colon sigmoide y parte del útero; y en el caso 4 , involucraba la cara posterior del útero y ligamento ancho, ocluía el fondo de saco posterior y se adhería al colon sigmoide y asas intestinales (Tabla 3 ).

Las características de implantación de la placenta permitieron su remoción, al practicar histerectomía en la pri- mera mujer, y anexectomía en la segunda, con sobrevivencia de éstas dos mujeres (Tabla 3).

Por el contrario, en el tercero y cuarto caso, fue necesario dejar la placenta in situ y, posteriormente, se administró metrotexate (MTX) (Tabla 3). En el caso 4 fue necesario dejar empaquetamiento de compresas para poder hacer hemostasia, y se efectuó una segunda laparotomía encontrando buena hemostasia, se retiraron las compresas y se dejó la placenta in situ. Estas dos últimas mujeres murieron; una por tromboembolismo pulmonar y la otra por sépsis, como causas inmediatas de muerte. En ninguno de los casos fue autorizada la necropsia.

El primero y el último caso llegaron al término con feto vivo, sin deformidades. En el primer caso, el recién nacido sobrevivió (Tabla 3 ) y fue dado de alta en buenas condiciones al igual que su madre. 
Tabla 3

INSERCION PLACENTARIA, MANEJO Y RESULTADOS

\begin{tabular}{|c|c|c|c|c|}
\hline \multicolumn{5}{|c|}{ Casos de embarazo abdominal } \\
\hline Variable & 1 & 2 & 3 & 4 \\
\hline Placenta & $\begin{array}{l}\text { Fondo } \\
\text { uterino }\end{array}$ & $\begin{array}{l}\text { Anexo } \\
\text { derecho }\end{array}$ & $\begin{array}{l}\text { Anexo izq. } \\
\text { Sigmoide } \\
\text { Utero }\end{array}$ & $\begin{array}{l}\text { Utero } \\
\text { Lig. ancho } \\
\text { Douglas } \\
\text { Sigmoide } \\
\text { Intestino }\end{array}$ \\
\hline Manejo & Histerectomia & Anexectomía & Hemostasia & $\begin{array}{l}\text { Empaque- } \\
\text { tamiento }\end{array}$ \\
\hline MTX & No & No & $\mathrm{Si}$ & Si \\
\hline Mujer & Sobrevivió & Sobrevivió & Murió & Murió \\
\hline Feto & Sobrevivió & Murió & Murió & Murió \\
\hline
\end{tabular}

\section{Discusión}

Si tenemos en cuenta que entre 1970 y 1993,0.15\% de los embarazos ectópicos diagnosticados en el HC fueron abdominales (1) y que según la literatura, el embarazo abdominal puede ocurrir con una frecuencia de entre 0.03 (2) y 1.3 (3) por 100 embarazos ectópicos, podemos considerar que en el HC hubo un "comportamiento epidémico" de embarazo abdominal entre 1993 y 1995, toda vez que ocurrieron 2.6 embarazos abdominales por cada 100 embarazos ectópicos (4).

Lo anterior nos lo confirman las tasas de incidencia de embarazo abdominal de 4.6/10.000 nacidos vivos entre 1993 y 1995 (4) que, comparativamente con las del período 197093 de 2.1/100.000 nacidos vivos (1), resultan inusitadamente altas para este hospital y mayores que las de muchos hospitales del mundo, aún cuando las tasas de incidencia de embarazo abdominal varian según la región, la época y el denominador utilizado. Por ejemplo, Beacham (1962) informó 1 embarazo abdominal por 3,337 nacimientos en el Charity Hospital de Nueva Orleans (5); Stafford y Raagan (1977) I por 7.931 nacimientos en el Indiana University Hospital (6); Mburra (1986), 1 en 3.259 partos en Tanzania (7); Atrash (1987), estimó que ocurre 1/9,174 nacidos vivos en Estados Unidos (8); y, más recientemente, Ahmed (1996) informó 1/5,000 partos en Sudán (9).

No hay datos colombianos disponibles sobre incidencia de embarazo abdominal durante el período descrito que nos permitan comparar con las cifras del HC. Según Jubiz (10), se habían publicado 32 casos de embarazo abdominal en Colombia, hasta 1960; y sólo hay dos publicaciones recientes (11-12) que describen la ocurrencia de casos de embarazo abdominal temprano en los hospitales de Sogamoso y Universitario de Cartagena, sin análisis de incidencia en esas instituciones. Pienso que lo anterior puede deberse a subregistro en los hospitales colombianos y a insuficiente número de publicaciones en Colombia relacionados con estos casos, más que a una baja incidencia de embarazo abdominal.

Es muy probable que el "brote epidémico" de embarazo abdominal observado en el HC refleje tanto un aumen- to en la incidencia como un mayor interés en la detección y análisis de los casos.

El aumento en la incidencia de embarazo abdominal en el HC es un hecho real y, además, ha sido un fenómeno mundial y acorde con el incremento en las tasas de embarazo ectópico durante las dos últimas décadas y se relaciona con la prevalencia creciente de los factores de riesgo y, principalmente, de enfermedades de transmisión sexual y enfermedad pélvica inflamatoria (13-14).

Nuestra publicación en la Revista Colombiana de Obstetricia y Ginecología, describiendo un caso de embarazo abdominal ocurrido en 1993, propició un estado de alerta entre el cuerpo de obstetras y residentes del hospital con una consecuente mejoría en los registros y especificidad en el diagnóstico. Además, personalmente estuve pendiente de la identificación de los casos, de su adecuada recolección, codificación y entrada a la base de datos en la sección de estadística del hospital y, finalmente, de su procesamiento, análisis y publicación, lo cual, obviamente, ha evitado el subregistro, tan común en nuestros hospitales.

La implantación peritoneal del óvulo en el abdomen puede ser primaria; pero en la mayoría de los casos corresponde a la interrupción de una gestación tubaria seguida de implantación secundaria en la cavidad abdominal (15). Según la definición clásica de Studderford (16), el primero y cuarto casos del HC fueron primarios, y el segundo y el tercero, secundarios.

Aproximadamente, el $80 \%$ de los embarazos abdominales terminan en el primero o segundo trimestre, y es infrecuente que el embarazo llegue al término y menos aún que sobreviva (9). El embarazo abdominal avanzado se encuentra más comúnmente en países en desarrollo asociado a las limitaciones propias de éstos para un diagnóstico temprano (9, 17-18). La literatura latinoamericana informa, cada vez con mayor frecuencia, sobre la ocurrencia de embarazos abdominales de término (19-26). El diagnóstico temprano es más común en países desarrollados, probablemente debido al más pronto acceso a control prenatal y a la mayor disponibilidad de ecografía (8). Sin embargo, el diagnóstico de embarazo abdominal temprano es difícil cuando en la laparotomía se encuentra hemoperitoneo y destrucción tubaria y, por tanto, usualmente se diagnostica "ectópico roto", sin especificar si se trataba o no de embarazo abdominal secundario, siendo ésta otra causa muy común de subregistro del embarazo abdominal, en nuestros hospitales.

Los factores de riesgo de embarazo abdominal son los mismos descritos para el embarazo ectópico e incluyen enfermedad pélvica inflamatoria por Chlamidya (27) y otras de transmisión sexual (28) inductores de la ovulación (29), cirugía tubaria para esterilización o reconstructiva (30), uso de DIU (31-32) y anticonceptivos con base en progestágenos solos (33). En nuestros casos no podemos ser categóricos en afirmar que no hubiesen factores de riesgo asociados al embarazo abdominal, puesto que la anamnesis relacionada con este asunto, por lo regular, no es muy exhaustiva y los datos no quedan consignados en la historia clínica. Sin em- 
bargo, cabría pensar en antecedentes de enfermedad pélvica inflamatoria en la mujer con VDRl reactiva. Los estudios publicados no informan sobre asociación de embarazo abdominal y $\mathrm{ACO}$ con base en estrógenos y progestágenos, por lo que podemos presumir que, en el caso 1, éste hallazgo es casual.

El embarazo abdominal rara vez se sospecha y usualmente se diagnostica durante una laparotomía exploradora. El dolor abdominal recurrente es el síntoma más frecuente; otros signos clínicos incluyen sangrado genital intermitente, movimientos fetales disminuidos o ausentes, malposición fetal y cérvix cerrado (34). En raras ocasiones la gestación evoluciona silenciosamente hasta llegar al término (21). La inducción fallida del trabajo de parto tras una aparente muerte fetal in útero debe considerarse un signo importante (3536). El dolor abdominal puede ser de variada intensidad y localización y conduce al médico a diagnósticos diferentes como sucedió con nuestros casos, así: el caso 1 presentó dolor desde el segundo trimestre de gestación y se trató como amenaza de parto pretérmino, las características del dolor en el caso 2 hicieron pensar inicialmente en infección de vías urinarias y, en el caso 4 , en que el dolor fue agudo en epigastrio y acompañado de hematemesis, se pensó en enfermedad ácido péptica.

El método más eficaz utilizado en la actualidad para el diagnóstico del embarazo abdominal es la ecografía. Los rasgos ecográficos de embarazo abdominal pueden ser sutiles y requieren un elevado nivel de sospecha para reconocerlos. Estos rasgos incluyen: 1) visualización del útero en forma separada del feto; 2) no visualización de la pared uterina entre el feto y la vejiga urinaria; 3 ) estrecha aproximación de las partes fetales a la pared abdominal materna; 4) situación excéntrica o presentación anormal del feto; y, 5) visualización del tejido placentario extrauterino (37). La ecografía se asocia con diagnósticos equívocos hasta en un $50 \%$ de los casos de embarazo abdominal (38); sin embargo, su uso incrementa la probabilidad diagnóstica en más de dos tercios de los casos (34). En nuestros casos la ecogratia fue de poco valor.

Cuando se sospecha embarazo abdominal, usualmente está indicada la intervención quirúrgica inmediata para disminuir el riesgo de muerte materna y fetal $(36,39)$.

Los sitios de implantación placentaria informados para embarazo abdominal primario incluyen el hígado (40), el bazo (41), el mesenterio intestinal, los vasos ilíacos y el diafragma (42), el fondo de saco de Douglas (43), la superficie serosa del útero (32), el epiplón (44-45) y el peritoneo parietal del flanco y fosa ilíaca izquierda (26).
El manejo de la placenta sigue siendo un motivo de debate. Muchos clínicos consideran que lo más apropiado es dejar la placenta in situ y cerrar el abdomen, dejando un drenaje retroperitoneal y administrar MTX para favorecer su reabsorción (40). Thompson (46) documentó casos en los cuales la placenta permaneció durante 13 años en la cavidad peritoneal sin problemas para la paciente. Otros recomiendan extirpar la placenta toda vez que sea posible (34). Ahmed and Fawzi (9) informan de 12 mujeres con embarazo abdominal avanzado en quienes se extrajo la placenta y todas sobrevivieron. Este proceder disminuye el riesgo postoperatorio de infección e íleus. En esta pequeña serie de casos del HC, la no extracción de la placenta se asoció a mayor morbi-mortalidad materna. Sin embargo, la placenta se dejó in situ cuando técnicamente fue imposible desprenderla sin ocasionar un grave sangrado.

La letalidad materna por embarazo abdominal en el HC fue del $50 \%$, siendo ésta una de las más altas cifras informadas recientemente. Un estudio en 20 mujeres árabes documentó una mortalidad materna del 20\% (47). Clark y Jhones (48) publicaron estimaciones de la mortalidad materna que variaron entre 4 y $10 \%$. Los datos más recientes son mucho más favorables. En una revisión de 10 casos (35) y en otra de 12 casos, en Sudán, (9) no se registraron muertes maternas.

La muerte materna está frecuentemente relacionada con sepsis, hemorragia y complicaciones intestinales resultantes de dificultades con la separación placentaria (35). En los casos del HC, las muertes se relacionaron con tromboembolismo trofoblástico y sépsis.

Nuestra mortalidad fetal del $75 \%$ es consistente con los datos de otros estudios que publican índices que varían entre 30 y $100 \%(9,47-49)$. Uno de los principales factores determinantes de la supervivencia fetal es el estado de las membranas fetales y, por el contrario, si se produce la ruptura de las membranas, el feto generalmente fallece en la cavidad abdominal en el curso de un breve período como consecuencia del distrés respiratorio (40). El embarazo abdominal debería al menos considerarse como posible causa de muerte fetal inexplicada durante el segundo y tercer trimestre (39).

El embarazo abdominal también se asocia con una alta incidencia $(35-75 \%)$ de niños de bajo peso y anomalías fetales, incluidas deformaciones faciales y articulares, tortícolis e hipoplasia de las extremidades (34). Por fortuna, en nuestro caso 1 el recién nacido sobrevivió y nació de buen peso, en excelentes condiciones y sin deformidades.

\section{BIBLIOGRAFIA}

1. Jaramillo EL., Castro JJ., Angel R. Embarazo ectópico y abdominal, Hospital de Caldas, 1970-1993. Rev. Col. Obst. Ginecol. 1995; 46: 67-69.

2. Hill GA., Herbert CM. Embarazo ectópico. In: Copeland LI, Jarrel IF, McGregor JA. Ginecología. Panam SA 1994; 237-254.
3. Breen JL.A 21 year survey of 654 ectopic pregnancies. Am. J. Obstet. Gynecol. 1970; 106: 1004-1019.

4. Jaramillo EL. Morbimortalidad materna en el Hospital de Caldas, Manizales. Colombia Médica 1997; 28: 77-84. 
5. Beacham WD., Hernquist WC., Beacham DW. Abdominal pregnancy at Charity Hospital in New Orleans. Am. J. Obstet. Gynecol. 1962: 84: 1257.

6. Stafford JC., Ragan WD. Abdominal pregnancy: review of current management. Obstet. Gynecol. 1977; 50: 548.

7. Mburra JSI., Mgaya HN. Advanced abdominal pregnancy in Muhimbili medical center; Tanzania. Int. J. Gynecol. Obstet. 1986; 24: $169-176$

8. Atrash HK., FriedeA., Hogue CJR. Abdominal pregnancy in the United Stats: frrequency and maternal mortality. Obstet. Gynecol. 1987: 69: 333-337.

9. Ahmed B., Fawzi HW. Advanced abdominal pregnancy in the developing countries. J. Obstet. Gynecol. 1996; 16: 400-406.

10. Jubiz HA. Embarazo ectópico. En: Botero UJ, Jubiz HA, Henao G, ed. Obstetricia y Ginecología. Cali, Bogotá; Carvajal SA, 2a reimpresión, $4^{\text {a }}$ edición, 1992.

11. González MA., Heredia MM. Embarazo abdominal. Rev. Col. Obst. Ginecol. 1992; 43: 70-71.

12. Herrera SF., Caro LG., TordecillaYY., TordecillaYM: Caso curioso: embarazo ectópico intraperitoneal. Tribuna Méd. 1995; 92 : $222-228$.

13. CDC Ectopic pregnancy, United States, 1988-1989. MMWR 1992: 41: 591-594.

14. Makinen JI. Increase of ectopic pregnancy in Finland. Combination of time and cohort effects. Obstet and Ginecol 1989; 73:21-24.

15. Little KJ, Green MM. Abdominal gestation. J Emergency Med 1995; 13: 195-198.

16. Studder ford WE Primary peritoneal pregnancy. Am. J. Obstet. Gynecol. 1942; 44: 487-491.

17. Alto W. Is there a greater incidence of abdominal pregnancy in developing countries? Report of four cases. Med JAust 1989; 151: 412-414.

18 Hallatt JG, Grove JA. Abdominal pregnancy: a study of twenty one consecutive cases. Am. J. Obstet. Gynecol. 1985; 152: 444-449.

19. Cocozzella G., Plastino E., Uranga A., Granillo JJ., González ME. Embarazo extrauterino avanzado. Rev. Fac. Cienc. Med. Plata $1985 ; 8: 22-28$.

20. Díaz BJ., D-Ambrosio M., Figueroa de Peña B. Embarazo abdominal a término con feto vivo: reporte de un caso. Rev. Obstet. Ginecol. Venezuela 1985; 45: 220-223

21. Nahoum JC. Gravidez abdominal de termo com feto vivo e euplástico: apresentacao de um caso a estudo da literatura brasileira. Femina 1989; 17: 629-638.

22. Reyes FS., Manzano OBR., Puntonet R., Trujillo M., Chepapcioglu M., Ramírez DV., Díaz MO. Embarazo abdominal (frecuencia en nuestro medio). Rev. Cuban Obstet. Ginecol. 1989; 15: 233-240.

23. Schutt DSG., Wilke S., Uberti EH., Costa PL. Gravidez abdominal a termo, com o feto vivo pensando mais de 2500 gr: relato de um caso. Rev. Bras. Ginecol. Obstet. 1989; 11: 184-186.

24. Cordero IR., Garcia RC., AndérezVO., Morales LME. Embarazo abdominal: presentación de 3 casos con fetos vivos a término. Rev. Cuban Obstet. Ginecol. 1991; 17: 69-72.

25. Lastra LA., Ruiz BJA., Jiménez BEA., Manrique OLA. Embarazo abdominal con supervivencia fetal: presentación de dos casos. Ginecol. Obstet. Méx. 1993; 61: 348-350.

26. Alencar JCA., Carvalho NRH., Alencar MF., Seabra MC., Arrais RBM., Almeida FML., Oliveira FCH. Gravidez abdominal avancada com feto vivo: diagnóstico ecográlico e evolucao perinatal. Rev. Bras. Ginecol. Obstet. 1994; 16: 43-48.

27. Chow JM., Yonekura ML., Richwald GA., Greenland S., Sweet RL., Schachter J. The association between Chlamydia trachomatis and ectopic pregnancy. JAMA 1990; 263: 3164.-

28. Westrom L. Effect of acute pelvic inflammatory discase on fertility. Am. J. Obstet. Gynecol. 1975; 121: 707.

29. Gemzell C., Guillome J., Wang DF. Ectopic pregnancy following treatment with human gonadotropins. Am. J. Obstel. Gynecol. 1982; 143: 761

30. Wolf GC., Thompson NJ. Female sterilization and subsequent ectopic pregnancy. Obstet. Gynecol. 1980; 55: 17.

31. Marchbanks PA., Annegers JF., Coulam CB.. Strathy JH.. Kurland LT. Risk factors for ectopic pregnancy: a populationbased study. JAMA 1988;259: 1823.

32. Kasby C., Krins A. Primary peritoneal pregnancy in association with intrauterine contraceptive devices: two case reports. Brit. I. Obstet. Gynecol. 1978; 85: 794-795.

33. Bergsjo P., Langengen H., Aas J. Tubal pregnancies in women using progestagen-only contraception. Acta Obstet. Gynecol. Scand. 1974; 3: 377.

34. Rock JA. Embarazo ectópico. In: Thompson ID, Rock JA. Te Linde Ginecología quirúrgica. 7a. ed. Panam SA 1993; 382-405.

35. Delke I., Veridiano NP., Tancer MD. Abdominal pregnancy: review of current management and addition of ten cases. Obst. Gynecol. 1982; 60: 200.

36. Golland IM. A fatal maternal outcome after delayed delivery in a midtrimester abdominal pregnancy. Am. J. Obstet. Gynecol. 1985 153: 676-677.

37. Allibone GW., Fagan CJ., Porter SC. The sonographic features of intra-abdominal pregnancy. J. Clin. Ultrasound. 1981; 9: 383.

38. Costa SD., Presley J., Bastert G. Advanced abdominal pregnancy Obst. Gynecol. Surv. 1991; 46: 515-525.

39. York AC., Rettenmaier MA.An unusual complication of delayed management in a case of fetal death. Am. J. Obstet. Gynecol. 1984; 150: 101-102.

40. Mitchell RW., Teare AJ. Primary hepatic pregnancy: a case report and review. South African Medical J 1984; 65: 220.

41. Hubert DE., Martin SD., Orlay G. A case report of splenic pregnancy. Australian and New Sealand J Surg 1984; 54: 81-82.

42. Norenberg DD., Gundersen GH., Janis GF., Gundersen Al. Early pregnancy on the diaphragm with endometriosis. Obstet. Gynecol. 1977; 49:620-622.

43. Jhonson AG. Primary peritoneal pregnancy. Brit. Med. J. 1968; 4: 96-97.

44. Acker D., Jenson AB., Tenn GK. Abdominal pregnancy with intrauterine device in-situ. Obstet Gynecol 1973; 42: 36-39.

45. Sharara HA., Saad FA., Mohsen NA. Primary abdominal ectopic Pregnancy. J. Obstet. Gynecol. 1997; 17: 313-315.

46. Thompson LR.Abdominal pregnancy at term with later removal of placenta. Am. J. Surg. 1966; 111: 272.

47. Rahamn MS.,Al-Suleiman SA., RahmanAl-Sibai MH.Advanced abdominal pregnancy. Obstet. Gynecol. 1982; 59: 366.

48. Clark JF, Jones SA. Advanced ectopic pregnancy. J. Reprod. Med. $1975 ; 14: 30$

49. Martin JN Jr, Sessums JK., Martin RW. Abdominal pregnancy: current concepts of management. Obstet Gynecol. 1988; $71: 549$. 